\title{
Within-person variability in men's facial width-to-height ratio
}

Robin S. S. Kramer

Background. In recent years, researchers have investigated the relationship between facial width-to-height ratio (FWHR) and a variety of threat and dominance behaviours. The majority of methods involved measuring FWHR from 2D photographs of faces. However, individuals can vary dramatically in their appearance across images, which poses an obvious problem for reliable FWHR measurement. Methods. I compared the effect sizes due to the differences between images taken with unconstrained camera parameters (Studies 1 and 2) or varied facial expressions (Study 3 ) to the effect size due to identity, i.e., the differences between people. In Study 1, images of Hollywood actors were collected from film screenshots, providing the least amount of experimental control. In Study 2, controlled photographs, which only varied in focal length and distance to camera, were analysed. In Study 3, images of different facial expressions, taken in controlled conditions, were measured. Results. Analyses revealed that simply varying the focal length and distance between the camera and face had a relatively small effect on FWHR, and therefore may prove less of a problem if uncontrolled in study designs. In contrast, when all camera parameters (including the camera itself) are allowed to vary, the effect size due to identity was greater than the effect of image selection, but the ranking of the identities was significantly altered by the particular image used. Finally, I found significant changes to FWHR when people posed with four of seven emotional expressions in comparison with neutral, and the effect size due to expression was larger than differences due to identity. Discussion. The results of these three studies demonstrate that even when head pose is limited to forward facing, changes to the camera parameters and a person's facial expression have sizable effects on FWHR measurement. Therefore, analysing images that fail to constrain some of these variables can lead to noisy and unreliable results, but also relationships caused by previously unconsidered confounds. 


\section{Within-person variability in men's facial width-to-height ratio}

2

3 Robin S. S. Kramer ${ }^{1}$

4

$5 \quad{ }^{1}$ Department of Psychology, University of York, York, UK

6

7 Corresponding author:

8 Robin $\mathrm{Kramer}^{1}$

9 Department of Psychology, University of York, York, YO10 5DD, UK

10 Email address: remarknibor@gmail.com 


\section{Abstract}

12

13 Background. In recent years, researchers have investigated the relationship between facial

14 width-to-height ratio (FWHR) and a variety of threat and dominance behaviours. The majority of 15 methods involved measuring FWHR from 2D photographs of faces. However, individuals can 16 vary dramatically in their appearance across images, which poses an obvious problem for reliable 17 FWHR measurement.

18 Methods. I compared the effect sizes due to the differences between images taken with 19 unconstrained camera parameters (Studies 1 and 2) or varied facial expressions (Study 3) to the 20 effect size due to identity, i.e., the differences between people. In Study 1, images of Hollywood 21 actors were collected from film screenshots, providing the least amount of experimental control. 22 In Study 2, controlled photographs, which only varied in focal length and distance to camera, 23 were analysed. In Study 3, images of different facial expressions, taken in controlled conditions, 24 were measured.

25 Results. Analyses revealed that simply varying the focal length and distance between the camera 26 and face had a relatively small effect on FWHR, and therefore may prove less of a problem if 27 uncontrolled in study designs. In contrast, when all camera parameters (including the camera 28 itself) are allowed to vary, the effect size due to identity was greater than the effect of image 29 selection, but the ranking of the identities was significantly altered by the particular image used. 30 Finally, I found significant changes to FWHR when people posed with four of seven emotional 31 expressions in comparison with neutral, and the effect size due to expression was larger than 32 differences due to identity. 
33 Discussion. The results of these three studies demonstrate that even when head pose is limited to 34 forward facing, changes to the camera parameters and a person's facial expression have sizable 35 effects on FWHR measurement. Therefore, analysing images that fail to constrain some of these 36 variables can lead to noisy and unreliable results, but also relationships caused by previously 37 unconsidered confounds. 
39

40

41

42

43

44

45

46

47

48

49

50

51

52

53

54

55

56

57

58

59

60

61

\section{Introduction}

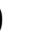

1

2

width-to-height ratio (FWHR; Weston, Friday, \& Liò, 2007) - and its predictive power when

considering a variety of human behaviours (for meta-analyses, see Geniole et al., 2015;

Haselhuhn, Ormiston, \& Wong, 2015). Although originally proposed as evidence that sexual selection played a role in shaping the human skull (Weston et al., 2007), researchers have subsequently found associations between FWHR and aggression, dominance, and threat behaviours in several domains (e.g., Carré \& McCormick, 2008; Stirrat \& Perrett, 2010; Wong, Ormiston, \& Haselhuhn, 2011). Interestingly, evidence suggests that FWHR is correlated with these behaviours, but it also predicts perceptions of faces when observers are asked to make judgements regarding these traits (e.g., Carré, McCormick, \& Mondloch, 2009; Stirrat \& Perrett, 2010). As a result, it has been argued that FWHR is an evolved cue of threat (Geniole et al., 2015).

Although FWHR was originally measured directly from skulls (Weston et al., 2007), almost all studies linking this ratio with behaviours have collected measurements from 2D photographs (e.g., Carré \& McCormick, 2008; Stirrat \& Perrett, 2010). Evidence suggests that measurements taken from images show high agreement with measures taken directly from the face (Kramer, Jones, \& Ward, 2012), although the nature of this as a suitable proxy for skull FWHR has not been determined. More importantly for the current work, photographs of the same individual can vary dramatically (Jenkins et al., 2011). Unconstrained images of a face vary in pose, expression, lighting, age, camera settings, and so on. Such variability can significantly decrease face matching performance, i.e., telling if two different images are of the 
62 same person (e.g., Megreya \& Burton, 2006, 2008). Indeed, this within-person variability

63 strongly argues against the idea that particular facial measures or distances underlie recognition

64 (Burton et al., 2015).

65 If facial measures vary across images of the same person, is it reasonable to assume a 66 reliable measure of FWHR can be obtained from a single 2D photograph? While lighting is 67 unlikely to affect measures of the face (other than shadows preventing accurate measurement), several other variables may significantly alter a person's apparent FWHR. Previous research suggests that FWHR decreases with age (Hehman, Leitner, \& Freeman, 2014; cf. Kramer, 2015), although this is not generally controlled for in the literature (but see Alrajih \& Ward, 2014). In addition, head pose (tilting upwards/downwards) has a sizable effect on FWHR obtained from photographs (Hehman, Leitner, \& Gaertner, 2013). This seems intuitive and, as a result, researchers have tended to include only images that are forward facing, i.e., looking directly at

74 the camera without any noticeable tilting or left-right rotation. In contrast, both facial expression and camera parameters appear less well considered. While many researchers have chosen to exclude images demonstrating expressions other than neutral (e.g., Zilioli et al., 2015), other researchers are less explicit in their inclusion criteria (Haselhuhn \& Wong, 2011) or acknowledge that non-neutral images were included (Carré \& McCormick, 2008). Regarding camera parameters, no FWHR research appears to have considered their effects. Interestingly, distance between the face and the camera, as well as the

81 camera's focal length, are known to alter facial appearance (Banks, Cooper, \& Piazza, 2014;

82 Harper \& Latto, 2001; Verhoff et al., 2008), with those photographed closer to the camera

83 appearing thinner and therefore having lower FWHRs (Bryan, Perona, \& Adolphs, 2012). 
In previous studies, researchers have either failed to consider, or have simply avoided

85 (through constraining photographic conditions), the potential influences of both facial expression

and camera settings. Importantly, in many situations where images are collected from real-world contexts (e.g., political races, sporting competitions, etc.), no such constraints can be imposed. In the current set of studies, I consider both influences on FWHR measurement. Through the calculation of effect sizes, I aim to determine how influential these factors might be, and hence whether researchers need to constrain or control for these effects in all future work.

To my knowledge, no previous research has included measurement of FWHR while systematically varying camera conditions or facial expressions. As such, it is difficult to make predictions regarding how these two factors may influence resulting measures. However, visual inspection of within-person photographic changes in facial expression suggests that these can produce significant alterations to FWHR. Therefore, I hypothesise that varying one's facial expression may have a larger effect on FWHR than differences between individuals. Similarly, with large changes to camera parameters (distance to camera in particular), we see noticeable FWHR differences (Harper \& Latto, 2001). Again, I would predict that these camera effects may be larger than the effect on FWHR due to differences between people's faces.

In the studies that follow, I focus on within-person variability in White men only (see Haselhuhn et al., 2015). The majority of research has established links between FWHR and various aggressive or competitive behaviours in men, but has generally failed to find such relationships in women (e.g., Carré \& McCormick, 2008; Haselhuhn \& Wong, 2011; cf. Lefevre et al., 2014). In addition, there may be significant differences in FWHR across ethnicities (Kramer, 2015). For these reasons, I investigated the effects of facial expressions and camera 
106 parameters on FWHR measures in White men, while avoiding any noise due to differences

107 between ethnicities, as the results would then be of the most relevance for the current literature.

108

109 Study 1 - Unconstrained camera parameters

110

In this study, I investigated the influence of variability in camera parameters on resulting

112 FWHR measures. Although all images were taken front-on and with a relatively neutral

113 expression, variables including the camera used, its focal length, and its distance to the subject

114 were unconstrained.

115

116 Materials \& Methods

Five White male Hollywood actors were selected based on their ages and their prolific film

119 appearances. For each actor, five films were chosen that were released while the actor was

120 between the ages of 30 and 35 years. This limited age range minimised the possibility that age

121 might influence any variability in FWHR both within and between actors (Hehman et al., 2014).

122 However, this time period does allow for potential fluctuations in body weight, perhaps required

123 for different roles, and this is known to influence FWHR (Coetzee et al., 2010; Geniole et al.,

124 2015). For each film, five screenshots were taken using VLC Media Player in which the actor

125 displayed a relatively neutral expression and was facing front-on to the camera (although gaze

126 was often not directed at the camera). Each screenshot was taken from a different scene in the

127 film, and no images included beards or glasses. As such, 25 images were collected for each actor. 
129 rotated using custom MATLAB software so that both pupils were aligned to the same transverse

130 plane. The same software was then used to measure the width (the horizontal distance between

131 the left and right zygions) and height (the vertical distance between the highest point of the upper

132 lip and the highest point of the eyelids) of each image. The FWHR was calculated as width

133 divided by height.

134 While every care was taken to include only images that were neutral and front-on, it must

135 be acknowledged that there remained some variability in these two parameters, in particular

136 where actors appeared relatively emotionally neutral but with their mouths open. As such,

137 emotional expression could also be considered to vary here, although this is investigated more

138 systematically in Study 3. Similarly, slight head rotations (both left/right and up/down) may also

139 be present. Importantly, these images were still within the range of head angles that have been

140 analysed in previous publications investigating real-world settings (e.g., Carré \& McCormick,

141 2008; Huh, Yi, \& Zhu, 2014; Kramer, 2015; Lewis, Lefevre, \& Bates, 2012; Loehr \& O’Hara,

142 2013; Welker et al., 2014; Wong et al., 2011), which are inherently less constrained than those

143 taken in the laboratory (e.g., Kramer et al., 2012; Özener, 2011).

144

145 Results

146

147 The variability in FWHR measures within and between actors can be seen in Fig. 1. It is

148 clear that there is significant variability within actors regarding their FWHRs. As such, ordering

149 these five actors in terms of FWHR would depend greatly on which particular image was

150 considered to represent each man. The effect sizes due to Identity (the differences between 
151 actors) and Image (the differences within actors) can be quantified using sums of squares (SS)

152 analyses (Jones \& Kramer, 2015; Morrison, Morris, \& Bard, 2013). By dividing the SS for each

153 factor (Identity, Image, Identity x Image) by the total SS, I obtained their $\eta^{2}$ effect sizes.

154 Although the five levels for the Identity variable made intuitive sense (each actor is a level), the

15525 levels for Image were less meaningful since there is no relationship between the orders of

156 images for each actor. Simply, there is no reason why Image 1 is the first image for John Cusack,

157 and this bares no relationship with Image 1 for Ben Affleck. Therefore, in order to obtain an idea

158 of the effect sizes for the two factors and their interaction regardless of image orders, SS were

159 calculated over 10,000 iterations, each time randomising the orders of images within actors. For

160 Identity, the $\eta^{2}$ was 0.41 (and was unaffected by the ordering of the images within actors since

161 this value only takes into account the differences between actors). For Image, the $\eta^{2}$ values over

162 all iterations were $M=0.12, S D=0.03$, and for the Identity x Image interaction, the $\eta^{2}$ values

163 were $M=0.48, S D=0.03$.

164 These analyses show that the differences between actors accounted for much more of the

165 variance in FWHR than the differences within actors (due to image variation). This may seem

166 surprising, and highlights the importance of identity differences, irrespective of the particular

167 image chosen, when measuring FWHR. The largest effect size was due to the interaction

168 between Identity and Image, suggesting that the differences between images depended on the

169 actor, and were not equivalent across actors. This is to be expected, given the random selection

170 of images - one actor's images may vary more than another's simply based on the particular

171 images/films used.

172 I repeated this analysis using only one film (and therefore five images) per actor in order to

173 better equate the variability in images within actors. With each actor limited to a shorter time 
174 period and a single role, the variability due to camera parameters remains while additional

175 changes due to weight fluctuations and character changes are minimised. Only the first film in

176 the set for each actor was considered, and effect sizes for Identity $(0.54)$, Image $(M=0.09, S D=$

$1770.05)$, and for the Identity x Image interaction $(M=0.36, S D=0.05)$ mirrored the pattern seen

178 above. However, Identity showed an even great effect here while the interaction effect decreased.

179 By removing the changes in FWHR due to differences across films for a given actor, which

180 perhaps have little equivalence in the real world, I found that FWHR was influenced more by

181 differences between people than within (due to particular images).

182 Another way to quantify the importance of considering (and potentially constraining)

183 camera parameters when selecting images is to model the rank correlation of the five actors

184 irrespective of which image was used. This method more closely resembles analyses in the

185 literature where FWHRs are correlated with behavioural measures, relying on the ordering (and

186 specific values) of the faces. For each iteration, I randomly selected two images for each actor

187 (from the 25 available). I then correlated the FWHRs for the five pairs of images, giving a

188 measure of agreement between the rankings of the actors irrespective of which images were

189 used. After 10,000 iterations, the distribution of rank correlations $(M=0.41, S D=0.42)$ showed

190 relatively low agreement. Even lower agreement was found when this analysis was repeated

191 using only images from the first film for each actor $(M=0.34, S D=0.45)$. Therefore, if

192 researchers fail to constrain camera parameters during image collection, there will be a sizable

193 effect on the orders of their actors according to FWHR.

194

195 Study 2 - Variation in focal length and distance to the camera

196 
198 influence on the apparent FWHR of a face. Next, I consider the effect of camera parameters on

199 FWHR under controlled laboratory conditions. By analysing images where focal length and 200 camera distance were systematically varied, I can determine their particular influence on FWHR 201 without additional noise due to head pose, emotional expression, etc.

202

203

Materials \& Methods

204

205

Photographs of 21 White adult men were taken from the Caltech Multi-Distance Portraits 206 database (Burgos-Artizzu, Ronchi, \& Perona, 2014). For each model, front-on images were taken using a Canon Rebel Xti DSLR camera at seven distances: 60, 90, 120, 180, 240, 360, and $480 \mathrm{~cm}$. Longer focal lengths were used with greater distances in order to equate the sizes of the images. Models were instructed to remain still and maintain a neutral expression throughout the procedure, which lasted 15-20 seconds. No images included beards or glasses. As above, images were rotated using custom MATLAB software so that both pupils were aligned to the same transverse plane, and then FWHRs were measured.

214 Results

As in Study 1, SS analyses were carried out on the FWHR values in order to quantify the 217 effect sizes due to Identity (the differences between models), Distance (the differences due to the 218 distance from the camera and its focal length), and their interaction. The results showed that 219 Distance $\left(\eta^{2}=0.18\right)$ had a much smaller effect on FWHR than Identity $\left(\eta^{2}=0.80\right)$. This suggests 
220 that the particular camera distance-focal length combination had little effect on FWHR measures

221 relative to the general differences between models. Even so, camera distance did have a

222 statistically significant effect on FWHR, $F(6,120)=196.23, p<.001$, with FWHR increasing

223 with greater distance to the camera (Bryan et al., 2012). Interestingly, the effect size of the

224 Distance $\mathrm{x}$ Identity interaction was very small $\left(\eta^{2}=0.02\right)$, suggesting that the way the camera

225 distance and focal length altered FWHR was equivalent for all models.

226 In addition, I quantified the importance of considering (and potentially constraining)

227 camera distance and focal length when selecting images by modelling the rank correlation of the

22821 models irrespective of which image was used (see Study 1). After 10,000 iterations, the

229 distribution of rank correlations $(M=0.75, S D=0.08)$ showed high agreement, suggesting that if

230 researchers fail to constrain camera distance and focal length, there will only be a limited effect

231 on the orders or rankings of their models according to FWHR.

232

233 Study 3 - Variation in facial expression

234

235 In addition to the influence of camera parameters on FWHR measurement, another source

236 of within-person variability comes from facial expressions. The same face posing different

237 expressions may significantly alter FWHR. In general, researchers utilise neutral expressions and 238 exclude all others (e.g., Welker et al., 2014), but as yet, there has been no investigation into how 239 expressions may systematically alter FWHR measurement. 
244 (Langner et al., 2010). For each model, front-on images of eight emotional expressions (based on

245 the Facial Action Coding System; Ekman, Friesen, \& Hager, 2002) were included: angry,

246 contemptuous, disgusted, fearful, happy, neutral, sad, and surprised. No images included beards

247 or glasses. As above, images were rotated using custom MATLAB software so that both pupils

248 were aligned to the same transverse plane, and then FWHRs were measured. See Fig. 2 for 249 examples.

250

251 Results

252

253

As in Study 1, SS analyses were carried out on the FWHR values in order to quantify the

254 effect sizes due to Identity (the differences between models), Expression (the differences due to 255 posed expression), and their interaction. The results showed that Expression $\left(\eta^{2}=0.58\right)$ had a 256 much larger effect on FWHR than Identity $\left(\eta^{2}=0.31\right)$. This suggests that the particular 257 expression a person wears has a large influence on their FWHR, and can alter the rankings of a 258 set of models. Interestingly, the interaction between these two factors was relatively small $\left(\eta^{2}=\right.$ 259 0.11), suggesting that particular expressions alter the FWHRs of models in similar ways. For 260 example, disgusted expressions may systematically increase FWHR measures across models 261 while surprised expressions decrease them.

262 By carrying out a repeated measures ANOVA, treating Expression as an 8-level factor that 263 varied within models, I was able to investigate which expressions significantly altered FWHRs in 264 comparison with a baseline neutral expression. As expected, I found a significant effect of 
265 Expression, $F(7,133)=103.44, p<.001$. The results of pairwise comparisons between the

266 neutral expression and the remaining seven expressions are illustrated in Fig. 3.

267 As Fig. 3 shows, models posing with disgusted or happy expressions significantly

268 increased their FWHRs in comparison with neutral (both $p s<.001$ ). In contrast, posing with a

269 fearful or surprised expression significantly lowered their FWHRs (both $p \mathrm{~s}<.001$ ). The

270 remaining three expressions had no significant effect on FWHR (all $p \mathrm{~s}>.05$ ).

271 In addition, I quantified the importance of considering (and potentially constraining) facial

272 expression when selecting images by modelling the rank correlation of the 20 models

273 irrespective of which image was used. After 10,000 iterations, the distribution of rank

274 correlations $(M=0.23, S D=0.20)$ suggests that if researchers fail to constrain expression during

275 image selection, there will be a sizable effect on the orders of their models according to FWHR.

276

277 Discussion

278

279

Across three studies, I investigated the influences of camera parameters and facial

280

expressions on FWHR measurement. While within-person variability can have a substantial

281

effect on FWHR measurement, this was not always the case.

282

The results of Study 1 suggest that failing to constrain camera parameters, or indeed the camera used, may not be as detrimental to a study's design as one might predict. Differences between identities accounted for a larger proportion of the variation in FWHR than differences within identities (across images). This identity effect became larger still, relative to withinperson image differences, when images were limited to only one film per actor, which might be considered more comparable to the variation one might expect in everyday faces. However, 
288 correlation analyses highlighted the substantial effect within-person differences could have on

289 the ranking or ordering of faces, an important issue for the majority of articles on this topic.

290 Therefore, collecting images taken by different cameras using different settings (in contrast with

291 the more constrained parameters of Study 2) will add substantial noise to any potential FWHR-

292 behaviour relationship, or on occasion, may even lead to the detection of spurious relationships if

293 these factors are confounded with the variables under investigation. For example, if I were to

294 compare the FWHRs of Democratic and Republican candidates in the US, and these two political

295 parties utilised two different photographers, it may be that the differing camera set-ups result in

296 apparent FWHR differences. Indeed, two sets of White men of approximately the same age,

297 taken using different camera set-ups, produced significantly different FWHRs in previous work

298 (Study 1 vs. Study 2 in Kramer et al., 2012).

299 Here, only five actors were included in Study 1 and so the specific effect sizes may alter

300 with a larger sample. Of course, one could also collect more images for each actor. The

301 important result is not the values themselves but the relative sizes of the effects. Indeed, the

302 FWHR values illustrated in Fig. 1 are comparable with those obtained in previous studies (e.g.,

303 Kramer et al., 2012; Özener, 2011). It is clear that even when relatively neutral, front-on images

304 are used, there can be large variation in FWHR for a single face.

305 While every care was taken to constrain the images used in Study 1, slight head rotations

306 and expressions may have been present. For example, it can be difficult to detect, and therefore

307 control, up/down head tilt in two-dimensional images. Previous research has demonstrated that

308 people may tilt their heads in order to affect perceived FWHR and appear more intimidating

309 (Hehman et al., 2013). In order to control for the noise in FWHR measurement due to head 
310 position, researchers might utilise three-dimensional imaging where possible (e.g., Kramer et al., 311 2012).

312 Study 2 showed that the distance to camera itself, along with alterations to focal length,

313 have only a relatively small effect on FWHR in comparison with between-subject differences.

314 This was also demonstrated when I considered the ranking or ordering of identities by FWHR.

315 Therefore, these factors appear less important when compared with the more unconstrained 316 images used in Study 1. Unfortunately, because camera distance and focal length were both 317 allowed to vary in the particular image set used, further research is needed in order to separate 318 out the influences of these two parameters.

319 Interestingly, I found only a small effect of the interaction between camera parameters and 320 identity in Study 2, suggesting that increasing the camera distance and focal length alters FWHR 321 consistently across different people. Indeed, this result has meant that computer scientists have 322 found some success in estimating camera distance using photographs of unknown people 323 (Burgos-Artizzu et al., 2014; Flores et al., 2013).

$324 \quad$ In Study 3, I found that FWHR changed substantially across different expressions.

325 Therefore, as researchers have already implicitly assumed, it is important to keep this variable 326 constrained when collecting image sets. However, the current results also suggest a caveat - only 327 four of the seven expressions investigated here significantly differed from neutral. As a result, 328 angry, contemptuous, and sad facial expressions may not require exclusion during image 329 collection (assuming the majority of images are neutrals). Importantly, happy expressions (i.e., 330 smiles) produced significantly larger FWHRs and these are the expressions that tend to appear 331 most in photographic sets (given that people often smile unless instructed otherwise). Therefore, 332 inclusion of these images may lead to potentially spurious results. For example, the recent 
333 controversy surrounding sexual dimorphism in FWHR (e.g., Kramer et al., 2012; Özener, 2011)

334 could be unintentionally affected if expression was not tightly constrained during photography,

335 given that women tend to smile more than men in various situations (LaFrance, Hecht, \& Paluck, 336 2003). Interestingly, smiling faces are also perceived as more competitive (Mehu, Little, \&

337 Dunbar, 2008), which fits well with research whereby faces with larger FWHRs are perceived as 338 more dominant, aggressive, etc. (e.g., Stirrat \& Perrett, 2010).

339 Facial height in the current work was measured as the distance from the highest point of

340 the upper lip to the highest point of the eyelids (Kramer, 2015; Kramer et al., 2012; Stirrat \&

341 Perrett, 2010). It is worth mentioning that other researchers have instead chosen to use the brow

342 as the upper boundary (e.g., Carré \& McCormick, 2008; Özener, 2011). It may be that facial

343 expressions have an even larger effect on FWHR measures if the brow is used, given the sizable

344 shift in the position of the eyebrows for a number of expressions (Ekman et al., 2002).

345 The results presented here are derived from White male faces only. As discussed in the

346 Introduction, the majority of findings to date regarding FWHR and its associations with

347 behaviours are in White men. However, there is no a priori reason to believe camera

348 manipulations or changes to facial expression have different sizes of effects in women or other

349 ethnicities. Of course, expressions may alter women's faces in systematically different ways

350 because their face shape, and hence the way they pose expressions, differs from men. As such, I

351 invite future researchers to address this question.

352 The current work focusses on potential issues when measuring FWHR from photographs,

353 given its variability across different images of the same individual. These issues are also relevant

354 when considering the broader topic of social trait inferences. Recently, researchers have been

355 investigating how different images of the same face can produce widely varying social 
356 perceptions of that individual (Jenkins et al., 2011). Foreseeing the work presented here,

357 Todorov and Porter (2014) noted that even invariant facial characteristics like FWHR are

358 susceptible to image variation. Combining these topics, there is evidence to suggest that

359 inferences more likely to be based on static cues (like FWHR) may vary less across images of

360 the same face in comparison with inferences based more on dynamic cues like muscle

361 movements (Hehman, Flake, \& Freeman, 2015). This growing body of research demonstrates

362 how image variation can have repercussions for several areas of study.

363

\section{Conclusions}

365

366

The current set of studies explores the importance of considering both camera parameters and facial expressions when investigating FWHR. With increasing numbers of researchers downloading images from the Internet in order to explore real-world contexts (e.g., politicians, presidents, professional fighters, football players), the ability to control these factors may be lost.

370 Critically, one must then question whether it is even meaningful to compare images where the

371 camera set-up varies across individuals, for example. To date, there has been no experimental

372 consideration of this particular factor to my knowledge. In conclusion, I recommend that future

373 researchers consider whether both camera parameters and facial expressions can be constrained,

374 and indeed need to be constrained, during image collection before undertaking real-world 375 investigations.

376

377 Acknowledgments

378 
I thank Alex Jones and Renée Lefebvre for comments on the manuscript.

380

381

\section{References}

382

Alrajih S, Ward J. 2014. Increased facial width-to-height ratio and perceived dominance in the

Banks MS, Cooper EA, Piazza EA. 2014. Camera focal length and the perception of pictures. Ecological Psychology 26:30-46.

387

388

389

390

391

392

393

394

395

396

397

398

399

400

401 faces of the UK's leading business leaders. British Journal of Psychology 105:153-161.

Bryan R, Perona P, Adolphs R. 2012. Perspective distortion from interpersonal distance is an implicit visual cue for social judgments of faces. PLOS ONE 7:e45301.

Burgos-Artizzu XP, Ronchi MR, Perona P. 2014. Distance estimation of an unknown person from a portrait. In: Fleet D, Pajdla T, Schiele B, Tuytelaars T, eds. Computer Vision ECCV 2014. Springer International Publishing, 313-327.

Burton AM, Schweinberger SR, Jenkins R, Kaufmann JM. 2015. Arguments against a configural processing account of familiar face recognition. Perspectives on Psychological Science $10: 482-496$

Carré JM, McCormick CM. 2008. In your face: Facial metrics predict aggressive behaviour in the laboratory and in varsity and professional hockey players. Proceedings of the Royal Society B 275:2651-2656.

Carré JM, McCormick CM, Mondloch CJ. 2009. Facial structure is a reliable cue of aggressive behavior. Psychological Science 20:1194-1198.

Coetzee V, Chen J, Perrett DI, Stephen ID. 2010. Deciphering faces: Quantifiable visual cues to weight. Perception 39:51-61. 
402 Ekman P, Friesen WV, Hager JC. 2002. Facial Action Coding System: The manual. Salt Lake 403 City, UT: Research Nexus.

404 Flores A, Christiansen E, Kriegman D, Belongie S. 2013. Camera distance from face images. In: 405 Bebis G et al., eds. Advances in Visual Computing - ISVC 2013. Springer-Verlag, 513-522. 406 Geniole SN, Denson TF, Dixson BJ, Carré JM, McCormick CM. 2015. Evidence from meta407 analyses of the facial width-to-height ratio as an evolved cue of threat. PLOS ONE

408 10:e0132726.

409

410

411

412

413

414

415

416

417

418

419

420

421

422

423

424

Harper B, Latto R. 2001. Cyclopean vision, size estimation, and presence in orthostereoscopic images. Presence 10:312-330.

Haselhuhn MP, Ormiston ME, Wong EM. 2015. Men's facial width-to-height ratio predicts aggression: A meta-analysis. PLOS ONE 10:e0122637.

Haselhuhn MP, Wong EM. 2011. Bad to the bone: Facial structure predicts unethical behaviour. Proceedings of the Royal Society B 279:571-576.

Hehman E, Flake JK, Freeman JB. 2015. Static and dynamic facial cues differentially affect the consistency of social evaluations. Personality and Social Psychology Bulletin 41:11231134.

Hehman E, Leitner JB, Freeman JB. 2014. The face-time continuum: Lifespan changes in facial with-to-height ratio impact aging associated perceptions. Personality and Social Psychology Bulletin 40:1624-1636.

Hehman E, Leitner JB, Gaertner SL. 2013. Enhancing static facial features increases intimidation. Journal of Experimental Social Psychology 49:747-754.

Huh H, Yi D, Zhu H. 2014. Facial width-to-height ratio and celebrity endorsements. Personality and Individual Differences 68:43-47. 
425 Jenkins R, White D, Van Montfort X, Burton AM. 2011. Variability in photos of the same face. 426 Cognition 121:313-323.

427 Jones AL, Kramer RSS. 2015. Facial cosmetics have little effect on attractiveness judgments 428 compared with identity. Perception 44:79-86.

429 Kramer RSS. 2015. Facial width-to-height ratio in a large sample of Commonwealth Games $430 \quad$ athletes. Evolutionary Psychology 13:197-209.

431 Kramer RSS, Jones AL, Ward R. 2012. A lack of sexual dimorphism in width-to-height ratio in 432 433 White European faces using 2D photographs, 3D scans, and anthropometry. PLOS ONE 7:e42705.

434 435

LaFrance M, Hecht MA, Paluck EL. 2003. The contingent smile: A meta-analysis of sex differences in smiling. Psychological Bulletin 129:305-334.

436

437

438 $24: 1377-1388$

Lefevre CE, Etchells PJ, Howell EC, Clark AP, Penton-Voak IS. 2014. Facial width-to-height ratio predicts self-reported dominance and aggression in males and females, but a measure of masculinity does not. Biology Letters 10:20140729.

442 Lewis GJ, Lefevre CE, Bates TC. 2012. Facial width-to-height ratio predicts achievement drive 443 in US presidents. Personality and Individual Differences 52:855-857.

444 Loehr J, O’Hara RB. 2013. Facial morphology predicts male fitness and rank but not survival in 445 Second World War Finnish soldiers. Biology Letters 9:20130049.

446 Megreya AM, Burton AM. 2006. Unfamiliar faces are not faces: Evidence from a matching task. $447 \quad$ Memory \& Cognition 34:865-876. 
448 Megreya AM, Burton AM. 2008. Matching faces to photographs: Poor performance in

449 eyewitness memory (without the memory). Journal of Experimental Psychology: Applied

450 14:364-372.

451

Mehu M, Little AC, Dunbar RIM. 2008. Sex differences in the effect of smiling on social 452 judgments: An evolutionary approach. Journal of Social, Evolutionary, and Cultural

454

455

456

457 Psychology 2:103-121.

Morrison ER, Morris PH, Bard KA. 2013. The stability of facial attractiveness: Is it what you've got or what you do with it? Journal of Nonverbal Behavior 37:59-67.

Özener B. 2011. Facial width-to-height ratio in a Turkish population is not sexually dimorphic and is unrelated to aggressive behavior. Evolution and Human Behavior 33:169-173.

458

459

460

461

462

463

464

465

466

467

468

469

Stirrat M, Perrett DI. 2010. Valid facial cues to cooperation and trust: Male facial width and trustworthiness. Psychological Science 21:349-354.

Todorov A, Porter JM. 2014. Misleading first impressions: Different for different facial images of the same person. Psychological Science 25:1404-1417.

Verhoff MA, Witzel C, Kreutz K, Ramsthaler F. 2008. The ideal subject distance for passport pictures. Forensic Science International 178:153-156.

Welker KM, Goetz SMM, Galicia S, Liphardt J, Carré JM. 2014. An examination of the associations between facial structure, aggressive behavior, and performance in the 2010 World Cup association football players. Adaptive Human Behavior and Physiology 1:1729.

Weston EM, Friday AE, Liò P. 2007. Biometric evidence that sexual selection has shaped the hominin face. PLOS ONE 2:e710. 
470 Wong EM, Ormiston ME, Haselhuhn MP. 2011. A face only an investor could love: CEOs'

471 facial structure predicts their firms' financial performance. Psychological Science 22:1478$472 \quad 1483$.

473 Zilioli S, Sell AN, Stirrat M, Jagore J, Vickerman W, Watson NV. 2015. Face of a fighter:

474 Bizygomatic width as a cue of formidability. Aggressive Behavior 41:322-330.

475 


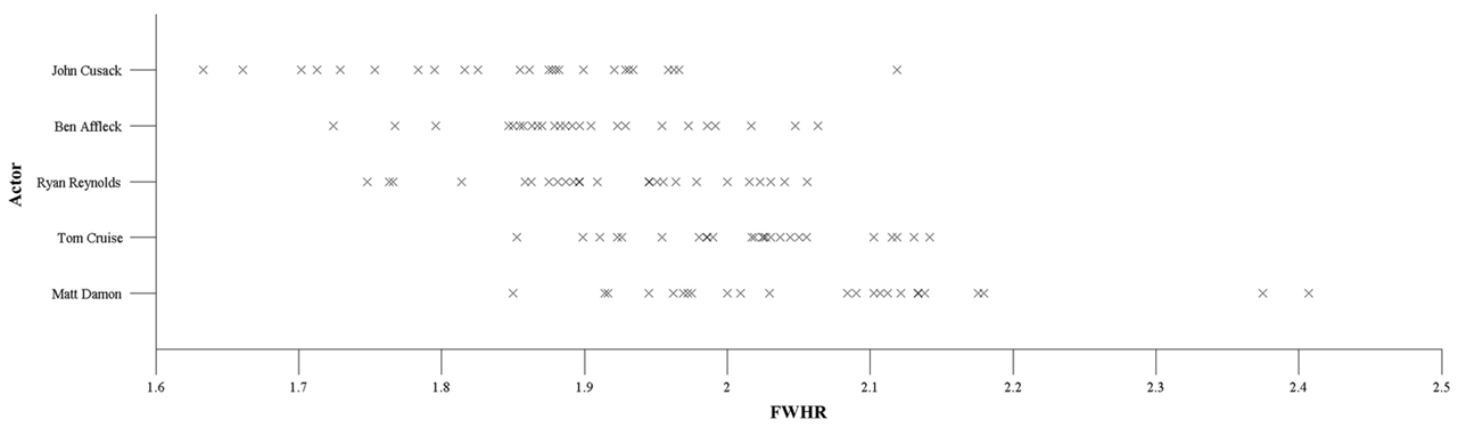

477 Figure 1. A scatterplot illustrating the within-person variability in FWHR for each actor. Each

478 cross is a different image.

479 


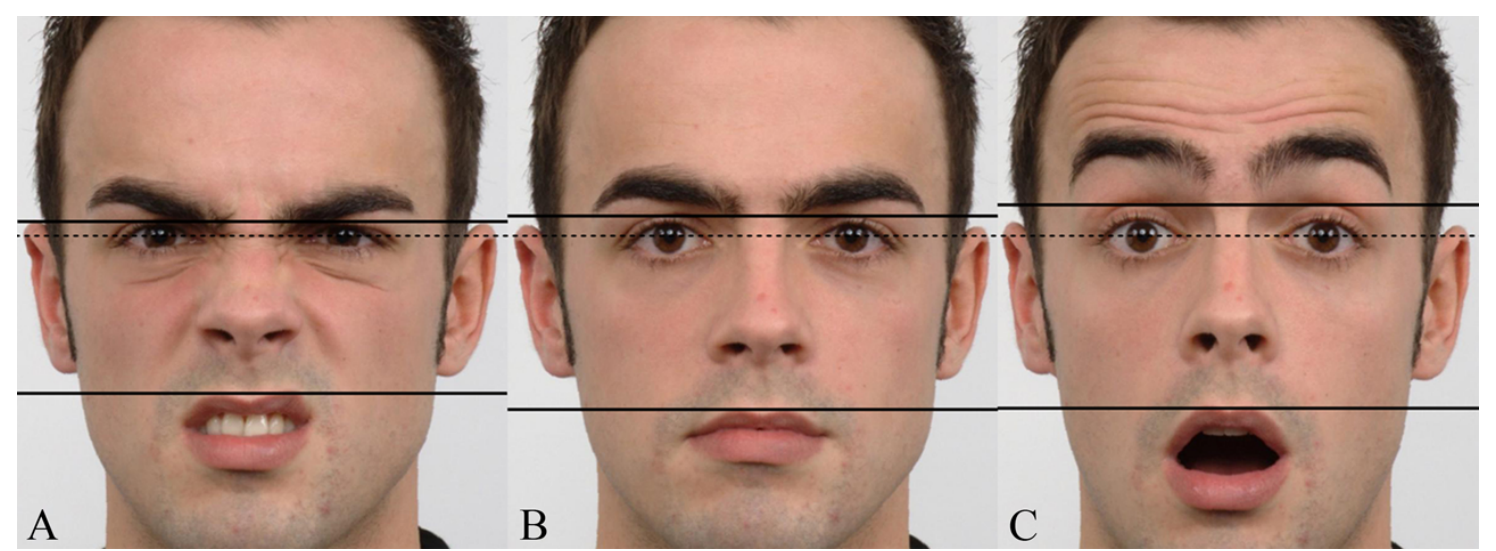

481 Figure 2. Example images (after rotation) from the Radboud Faces Database (reproduced with 482 permission). Horizontal lines depict the facial height for disgust (A), neutral (B), and surprise (C) 483 expressions. The dashed line illustrates that the pupils are level. 


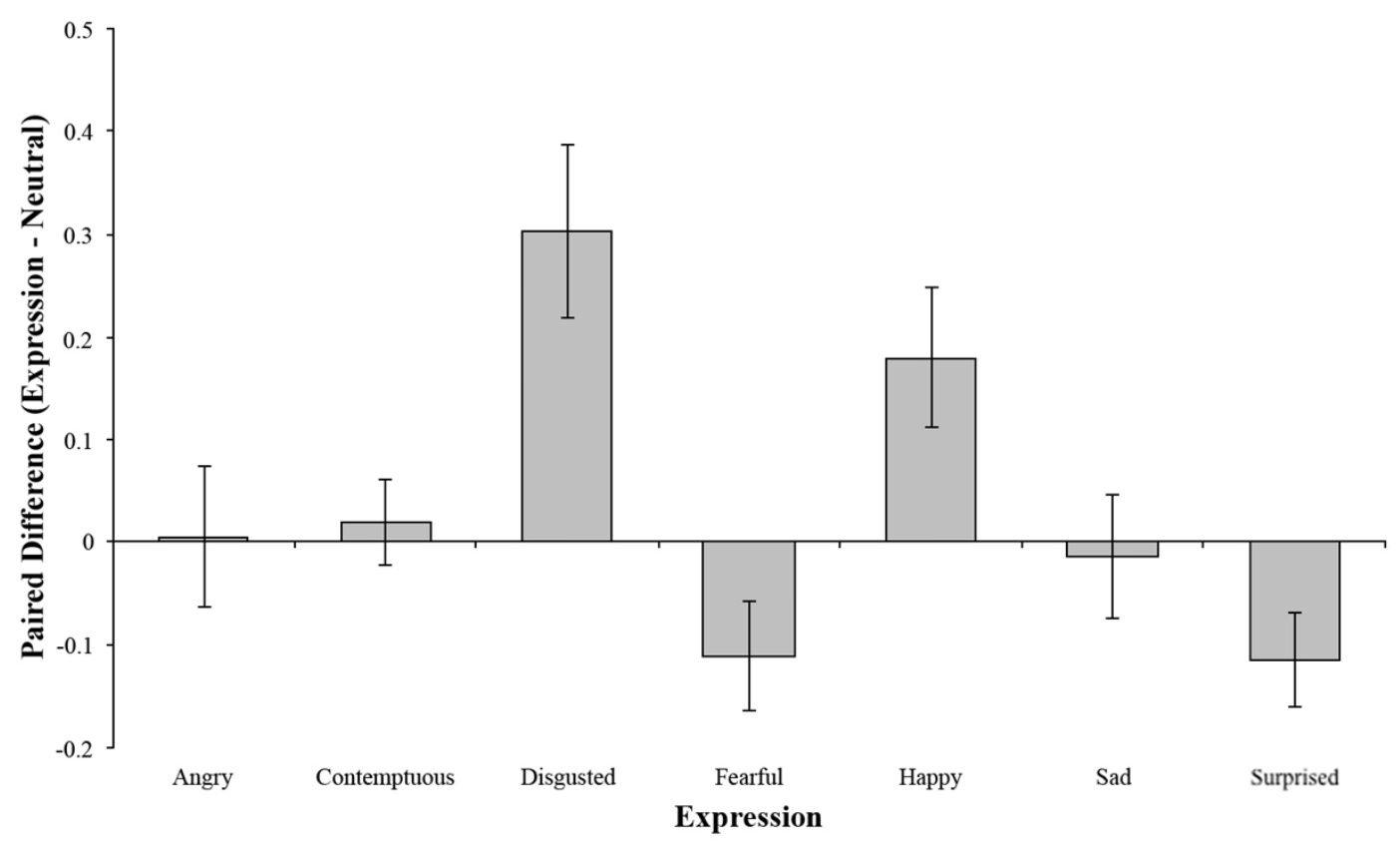

485

486 Figure 3. A bar chart illustrating the pairwise comparisons between the neutral expression and 487 the remaining seven expressions. The error bars represent the $95 \%$ confidence intervals of the 488 differences, adjusted for multiple comparisons using Bonferroni correction. As such, error bars 489 overlapping the zero line show no difference from neutral. 